\title{
La construcción discursiva de la juventud: lo general y lo particular ${ }^{1}$
}

\author{
Juan Carlos Revilla Castro \\ Universidad Complutense de Madrid. Facultad de Ciencias Políticas y Sociología \\ Departamento de Psicología Social \\ Campus de Somosaguas. 28223 Pozuelo de Alarcón (Madrid)
}

\section{Resumen}

En este artículo se desarrolla una descripción de los diferentes discursos que circulan en el ámbito de las ciencias sociales sobre la juventud en sus distintos aspectos. La mitificación de lo juvenil, el narcisismo, la juventud como producto social y como agente de cambio, la subcultura juvenil y su contestación, la transición a la vida adulta, la discriminación de la juventud, la búsqueda de la identidad y la diversidad de lo juvenil son los discursos que se analizan en sus contenidos, circulación social y consecuencias. Por último, se propone un modelo que recoge, desde los discursos analizados, proposiciones generalizables a toda la juventud actual como marco desde el que construir análisis particulares de los distintos segmentos juveniles.

Palabras clave: juventud, discurso, identidad, subcultura juvenil, cambio social.

\section{Abstract. The discursive construction of youth: the general and the particular}

In this article a description of the discourses about youth in its different aspects is developed. The mitification of youth, narcisism, youth as a social product and as agent of change, youth subculture and contestation, transition to the adult state, the discrimination of youth, the search for identity and the diversity of youth are the discourses that are analized in their contents, social circulation and consequences. Finally, we make a proposal of a model, gathering some general propositions from these discourses about youth adding some results of our research on young Spanish people.

Key words: youth, discourse, identity, youth subculture, social change.

\section{Sumario}

\section{Discursos sobre la juventud Bibliografía}

2. Organizando un discurso general sobre la juventud

1. Este artículo está basado en un capítulo de la tesis presentada por el autor en la Facultad de Ciencias Políticas de la Universidad Complutense de Madrid, por el Departamento de Psicología Social y bajo la dirección de la profesora Concepción Fernández Villanueva en 1996. 
La juventud en sus múltiples facetas se ha convertido en un área de estudio importante en ciencias sociales. Las investigaciones se han ocupado de los grupos de jóvenes cuyos comportamientos y actitudes son considerados preocupantes (subculturas juveniles, delincuencia juvenil, hinchas de fútbol) o se han interesado por dar un diagnóstico lo más acertado y completo posible acerca de la juventud del momento. En el caso español, la menor tradición subcultural hasta épocas recientes y el hecho de que las subculturas españolas parezcan reproducciones miméticas de lo que sucede en el mundo anglosajón, han conducido a un predominio de los estudios generales de juventud. Estos estudios, basados en encuestas realizadas ad hoc, se ocupan de todos los aspectos reseñables de la vida juvenil: familia, estudios, ocio, actitudes, costumbres, religión, vivien$\mathrm{da}$, asociacionismo, etc., con diferente énfasis según los intereses de la fundación o de la administración que encarga el estudio. Una parte importante siempre de estos estudios concierne a las actitudes de los jóvenes, en un intento de conocer lo que piensan y así saber si están en condiciones de ser adultos responsables, para, en caso contrario, actuar sobre ellos.

Se trata de una tendencia que proviene de los años sesenta, cuando el Instituto de la Juventud comenzó a interesarse por estudiar a la juventud del momento (ver Sáez Marín, 1995). Esta situación llega hasta nuestros días, con una proliferación de estudios nacionales, regionales, locales («monocultivo de estudios-encuesta»; Díaz, 1989), si bien es cierto que también se realizan cada vez más estudios sectoriales y cualitativos. Pero, por otro lado, son numerosas las publicaciones con intención científica que parecen basadas más en reflexiones personales del autor que en investigaciones propias o ajenas y que se convierten en potentes generadores de discursos. De esta forma, tenemos una situación harto confusa; mientras que, por un lado, abundan las reflexiones sin datos, por otro, son mayoría, salvo notables excepciones, las investigaciones sin ideas, con escasa labor interpretativa que conduzca al análisis en profundidad de los datos obtenidos.

Este inmenso trabajo científico es reflejo de la preocupación adulta por la juventud, una preocupación que tiene que ver con el papel que se otorga a los jóvenes como augurio del futuro que nos espera. Esta situación ha sido posible por la tendencia a pensar la juventud como un todo diferenciado respecto de los adultos, los cuales han de velar por los jóvenes como grupo y cuidar de que lleguen a ser adultos responsables que cumplan con el papel que les toque asumir. En definitiva, asegurarse de que la reproducción social se realice adecuadamente.

Este esfuerzo investigador ha ayudado a conocer, pero también a crear, una potente construcción social sobre la juventud en extremo poliforme y contradictoria. En este trabajo vamos a tratar de mostrar los diferentes discursos que circulan en el ámbito académico con su grado de penetración en el ámbito extracadémico, así como las consecuencias o derivaciones que tienen estos discursos para sus protagonistas, los jóvenes.

La simple realización de un inventario de discursos sobre la realidad juvenil es, en sí mismo, un instrumento que puede resultar útil para darnos cuen- 
ta de las interpretaciones que la sociología de la juventud ha sido capaz de aportar para el entendimiento de esta realidad tan compleja y difícil de conocer como es este polifacético grupo social. Supone la constatación de un «estado de la cuestión", a partir del cual podemos continuar nuestra labor científica. Y es esto lo que realizaremos más adelante, proponer un modelo de interpretación de la realidad juvenil en lo que tiene de común, dejando al margen las grandes diferencias internas. Esta comunalidad se basa en una situación estructural similar y en una construcción social sobre la juventud que todo el grupo soporta.

\section{Discursos sobre la juventud}

Antes de nada, será necesario realizar alguna consideración metodológica sobre el tipo de análisis que se va a realizar. Existen muchas formas diferentes de acometer un análisis de discurso, si bien en lo fundamental se distinguen en lo que se pretende extraer de los textos analizados: relaciones sociales, relaciones de poder, pautas de interacción, identificación de discursos o repertorios interpretativos, contenidos y procesos cognitivos, etc. La posición desde la que partimos en este análisis se apoya fundamentalmente en la escuela británica de análisis de discurso, que ha dado lugar a dos ramas diferenciadas en sus pretensiones y planteamientos (Widdicombe y Wooffitt, 1995): el grupo de Potter, Wetherell y Edwards, y el grupo de Parker y Burman.

En concreto, lo que vamos a tratar de realizar es la identificación en la literatura científica de una serie de discursos sobre la juventud, entendidos como «sistema coherente de significados acerca de un objeto determinado» (Parker, 1992, p. 10). Estos discursos son recursos sociales susceptibles de ser utilizados en cualquier argumentación o discusión de carácter científico, y pueden convertirse en recursos utilizables por cualquier miembro de la cultura en la medida en que se difundan y sean conocidos por la población.

El mayor problema que existe a la hora de intentar poner orden en la literatura sobre juventud es la gran variedad de discursos que es posible encontrar. Unos autores inciden sobre unos aspectos que no parecen importar a otros. Los niveles de discurso son igualmente heterogéneos. Por tanto, asistimos a una mezcla multiforme de proposiciones y argumentos más o menos sugerentes, más o menos plausibles.

La forma de diferenciar y aislar analíticamente unos y otros discursos ha pasado por identificar temas, preocupaciones, líneas de argumentación que parecen similares, con sus matices, por supuesto. De este modo, cada autor normalmente utiliza de hecho más de un discurso, articulándolo de una manera particular, si bien siempre en la medida en que cada discurso lo permite. Por tanto, los discursos de los que nos ocupamos se sitúan entre la explicación psicológica y la sociológica — prescindimos nítidamente de la biología y de los discursos biologicistas-, quizá más cerca de la segunda, y en todo caso ocupándonos del espacio propio de la psicosociología, la interacción social (Crespo, 1995, p. 129). 
En el fondo, lo que se está estudiando en estos discursos es el grado de agencialidad que hemos de conceder a la juventud como sujeto histórico y a cada joven en particular. Esto es, hasta qué punto tienen sentido las explicaciones que sitúan a la juventud como sujeto o como objeto, como agente o como producto de una sociedad. Quizá nuestra respuesta, como se verá más adelante, está más cerca de lo segundo que de lo primero, lo que sitúa a la agencia de los jóvenes más en el ámbito de su interacción cercana que en el marco de la sociedad global.

\section{Mitificación de lo juvenil}

Son numerosas en la literatura las alusiones a un discurso que incide sobre la mitificación de todo lo juvenil (e.g. Aranguren, 1982). Según este discurso, se manifiesta en nuestra sociedad una exaltación de todo lo joven. Es un culto al efebo, a la plenitud vital que los jóvenes experimentan y que los adultos y los mayores añoran. Como correlato de esta valoración, se produce una juvenilización de la sociedad (Moya, 1983), una apropiación de los adultos de los símbolos y los modos de los jóvenes (Lozano i Soler, 1994; Beltrán y otros, 1984). Por tanto, lo joven se convierte en criterio de éxito, en moda susceptible de ser generalizada por el resto de la sociedad (Arranz, 1982, p. 22).

Es éste un discurso que se encuentra muy presente en la sociedad en su conjunto, que toma forma en la añoranza de esa juventud perdida. No queda otra posibilidad que reivindicar para sí mismos un supuesto "espíritu juvenil», lo joven se convierte en un "estado de espíritu, una mentalidad" (Aranguren, 1982, p. 4). Los jóvenes interiorizan este discurso y se creen en la mejor época vital sin apenas haber vivido una pequeña parte de su vida (Revilla, 1998).

Estas consideraciones se han convertido en un lugar común a pesar de estar basadas en reflexiones y observaciones insuficientemente sistemáticas. Las interpretaciones que se pueden derivar de este discurso serán diferentes según en qué aspectos se base la juvenilización: estética, modo de vida, actitudes determinadas, etc. Además, siendo la juventud una categoría con importantes diferencias en su seno, habría que constatar de qué segmento juvenil proceden los elementos adoptados por los adultos.

Son escasas las explicaciones que se ofrecen para explicar este fenómeno de la mitificación de lo juvenil. Beltrán y otros (1984) plantean, a este respecto, que los adultos se vuelven hacia los jóvenes dada la posición que ocupan. Esta posición les sitúa cerca de la innovación social vertiginosa que experimenta nuestra sociedad y que conduce a los adultos a mirar hacia los jóvenes para no quedarse atrás. Como vemos, es una explicación que coloca a la juventud como un agente importante de cambio social (ver más adelante).

Quizá la consecuencia más importante de este discurso sea la creación de importantes expectativas para las personas que se encuentran o entran en esta etapa vital y que están en flagrante contradicción con la posición social subordinada que ocupan los jóvenes. 


\section{El hedonismo narcisista}

Frente al anterior discurso que ensalzaba lo juvenil, el discurso del narcisismo supone más bien una crítica para la juventud actual. Podemos distinguir tres repertorios diferentes pero muy relacionados: hedonismo, privatización y narcisismo propiamente dicho.

Por un lado, se afirma el hedonismo de la juventud, su valoración del placer como criterio moral (Arranz, 1982, p. 19). Además, existe un cierto énfasis en su espontaneidad e irracionalidad (Beltrán y otros, 1984, p. 193). Es, pues, una juventud materialista, que persigue la realización personal en términos de tener (Martín Serrano, 1992, p. 45) y consume activamente los productos del mercado juvenil (Durá Grimalt, 1987; Moya, 1983).

La juventud es igualmente conformista y descomprometida (Arribas y González, 1987), se desentiende de la política y de su papel transformador de la sociedad. Los jóvenes permanecen en el ámbito privado y no se ocupan de lo público como reacción a la desconfianza en las instituciones políticas. Esta tendencia a la privatización supone un cambio de valores: se prefiere la libertad privada a la igualdad (Martín Serrano, 1992, p. 47).

Por último, y como correlato de la privatización, asistimos a un fuerte narcisismo, que Beltrán y otros (1984) describen como interés por el autoexamen, preocupación por la realización personal y espíritu biológico consciente (p. 195).

La implantación de este discurso es importante. Está en los medios de comunicación, en los discursos de educadores, filósofos, padres, etc. Su presencia es tan fuerte que los propios jóvenes han de pronunciarse frente a él, negarlo o afirmarlo, no pueden permanecer al margen.

Tal construcción se fundamenta en una comparación implícita o explícita con otra generación juvenil, la que protagonizó las revueltas de los años sesenta. De hecho, parece que se ha construido un discurso que mitifica esa juventud como ejemplo de generación rebelde empeñada en la transformación de la sociedad. Ante tal mito, cualquier generación posterior parecerá conformista si no se producen acontecimientos similares. Quizá incluso fuera necesaria una deconstrucción del mito, ocuparse de las voces ocultas de esa generación, los segmentos de esa juventud que no participaron en las revueltas.

Este discurso manifiesta una preocupación por que la juventud no cumpla el papel que está llamada a desempeñar en su etapa adulta. Temor a los excesos hedonistas (sexo, drogas), a la falta de valores apropiados desde la moral adulta dominante, a la desimplicación en lo público. Pero este discurso sirve también para mantener a la juventud en una práctica minoría de edad: no están preparados para las responsabilidades adultas, no pueden ser aceptados como actores sociales plenos. Gil Calvo (1985, p. 15) se ha referido a algo parecido con la expresión "doble vínculo», pues mientras se les exige responsabilidades a los jóvenes, se les deniega la posibilidad de que las contraigan. 


\section{La juventud como producto histórico y/o social}

Este discurso pretende sustentar el carácter de la juventud, tal como la conocemos actualmente, como algo específico de nuestra sociedad occidental moderna, para lo cual se describen las características y la situación de la juventud en otros períodos históricos, pero también en otras culturas actuales. El trabajo de Philippe Ariès (1973) ha sido especialmente influyente en la expansión de este discurso. Según él, en el siglo XVII por juventud se hacía referencia en ese momento histórico a la etapa de plenitud vital, la primera parte de la época adulta. La juventud aparece a través de la prolongación de la adolescencia y el retroceso de la madurez, especialmente después de la Primera Guerra Mundial (ibídem, p. 54). Así se separa la juventud de la etapa adulta como este período vital de madurez física y dependencia material y afectiva. Posteriormente al trabajo de Ariès han proliferado estudios sobre jóvenes de otras épocas históricas, de entre los que cabe señalar el volumen dirigido por Levi y Schmitt (Historia de los jóvenes, 1995).

$\mathrm{La}$ antropología ha visto siempre la juventud como una de las posibles formas culturalmente determinadas de transición entre la niñez y la vida adulta. Así, se ha señalado la transición inmediata que caracteriza a las sociedades primitivas (Feixa, 1998).

Desde la sociología, se tiene por lugar común que la juventud aparece como fenómeno diferenciado después de la Segunda Guerra Mundial. Así, se relaciona con el período de expansión económica de los años cincuenta y sesenta, que produjo un excedente económico que hizo aparecer el mercado juvenil. La juventud sería, pues, algo exclusivo de las sociedades urbano-industriales (Zamora Acosta, 1990, p. 24). Bien es verdad que las condiciones de aparición de la juventud se crean con las transformación del feudalismo al capitalismo en las sociedades occidentales, lo cual produjo cambios en el papel de los jóvenes por la transformación de las instituciones que los acogían: familia, escuela, trabajo y ejército (ver Feixa, 1998).

Este discurso histórico parece haber penetrado en buena medida en el pensamiento cotidiano: se habla de una transición más inmediata en el pasado entre niñez y etapa adulta y de las diferencias entre la juventud de antes y la juventud actual. La mayor base para la extensión de este discurso se encuentra en las constantes comparaciones que realizan los progenitores entre su juventud y la de sus hijos y que no dudan en comunicar a éstos últimos.

Además, las pretensiones biologicistas de otro tipo de discursos son frenadas por este tipo de planteamientos. Las proposiciones solamente pueden plantearse como válidas mientras las condiciones de la juventud se mantengan parecidas. Pero no siempre se realizan las investigaciones del pasado y del presente para establecer divergencias y convergencias entre unos y otros momentos históricos con suficiente base empírica.

Absolutamente congruente con el discurso histórico es la idea de que la juventud es un producto de la sociedad en que vivimos (Corraliza, 1985), pues los problemas sociales se condensan o se reflejan en la juventud (Lozano i Soler, 
1994, p. 38). Así, se desresponsabiliza a los jóvenes de las caracterizaciones negativas que circulan sobre ellos, si bien no se niegan. Es ésta una de las estrategias que utilizan los jóvenes en su discurso cotidiano para desmarcarse del estereotipo negativo que sufren como categoría social, lo que da idea de la extensión de su circulación social (Revilla, 1998).

Pero este discurso convierte también a los jóvenes en sujetos necesitados de ayuda, protección o educación. Si no tienen responsabilidad sobre cómo son y lo que les pasa, serán los adultos los que habrán de intervenir cambiando la sociedad, pero sobre todo cambiando a los propios jóvenes (intervención social, programas de juventud, etc.).

\section{Juventud como agente de cambio social}

El discurso del cambio social es uno de los más extendidos en la sociología para explicar el papel que tiene la juventud en nuestra sociedad. Los precedentes de este discurso los podemos encontrar en Ortega y Gasset y Mannheim, como impulsores del estudio y la teorización sobre las generaciones, aunque es especialmente Ortega quien se encarga de otorgar a la juventud un papel histórico preponderante en el cambio de la sociedad, casi como sustituto de la clase social (Martín Criado, 1998).

El discurso de la juventud como agente de cambio se integra, aunque pueda parecer contradictorio, junto a la reproducción social. La reproducción social requiere de la aportación sucesiva de nuevas generaciones que se integren en la estructura en sustitución de las anteriores. La sociedad se ha de encargar de socializar y de ubicar en esa estructura a sus futuros integrantes (Allerbeck y Rosenmayr, 1977, p. 17). Pero la juventud es la encargada de aportar las innovaciones al integrarse en la estructura social. Por tanto, los jóvenes son protagonistas tanto de la reproducción como de la transformación social y se convierten en un sujeto histórico con entidad propia, una especie de «minoría activa» (Moscovici, 1979) que va ejerciendo su influencia en los diferentes ámbitos sociales.

Por tanto, los cambios sociales se han de condensar en la juventud, pues «las condiciones específicas de la juventud catalizan las crisis de cambio latentes en la sociedad» (Zárraga, 1985, p. 17). Además, la vitalidad juvenil implica ansias de cambio e innovación (García Rincón, 1994), incluso de revolución («juventud como clase revolucionaria», Aranguren, 1982, p. 5) y la juventud recibe y produce privilegiadamente los nuevos lenguajes (Cembrano, 1986, p. 165).

Según Beltrán y otros (1984), las nuevas generaciones ya no pueden apoyarse en la tradición para afrontar los nuevos problemas, pues no sirven. Por ello, los jóvenes han de crear sus propias respuestas, distanciándose de las pautas culturales obsoletas. Sin embargo, no siempre se logra la transformación de la sociedad, sea porque no se tenga la suficiente fuerza (ibídem), sea porque se opte por la adaptación y acomodación a la sociedad (Allerbeck y Rosenmayr, 1977 , p. 138). De ahí la posibilidad de hablar de juventudes más conformistas o más rebeldes y revolucionarias. 
El discurso del cambio está muy presente fuera del ámbito académico. El interés que se presta a «lo joven» es signo de que pensamos el futuro a través de ellos (Lozano i Soler, 1994, p. 41). Por tanto, se siente esperanza cuando la caracterización de la juventud es positiva (e.g. solidarios, comprometidos) y temor cuando es negativa (e.g. hedonistas, sin valores).

Situar en la juventud la posibilidad e incluso la responsabilidad del cambio social tiene, cuando menos, dos consecuencias claras. Por un lado, se exige a la juventud que cumpla el papel de innovadora, transgresora. Pero la innovación no debe cuestionar el sistema en su conjunto, ha de ser un cambio «incruento». En palabras de Allerbeck y Rosenmayr (1977, p. 132), lo que se espera de los jóvenes es una conformidad divergente, una desviación en la dirección adecuada. Por otro lado, el mundo adulto elude así la responsabilidad por la mejora de la sociedad que recae exclusivamente sobre la juventud.

En cualquier caso, la afirmación de la juventud como sujeto de cambio es tan «evidente» que no ha sido apenas investigada realmente. Los estudios no llegan a plantearse de qué manera la diversidad de las tendencias juveniles inciden sobre el cambio social —lo impide, lo alimenta, el cambio social es diferenciado según los grupos y ámbitos sociales. Pero tampoco parece cuestionarse si hay relación entre las características de una juventud y lo que serán después cuando alcancen el estatus adulto. La suposición clave es que existe una relación unívoca: según sean los jóvenes, así serán los adultos. Pero podría ser de otro modo, como se afirma de los jóvenes de la generación del 68, pues tiene poco que ver lo que fueron y lo que son - se han "convertido" al sistema. Además, podría ser que el hecho de que la juventud se vea como una etapa totalmente diferenciada de la etapa adulta refuerce la tendencia a vivirla de un modo poco relacionado con lo que será la posterior identidad adulta.

\section{La contestación juvenil}

El discurso de la contestación se interesa especialmente por los valores de los jóvenes, que se han convertido en materia de discusión predilecta. Esto tiene mucho que ver con el hábito de ver el futuro a través de los jóvenes y suponerles como agentes de cambio. Pero mientras en el narcisismo se veía en la juventud como portadora de unos valores negativos, en este discurso se sitúan en la juventud una serie de valores diferentes de los valores adultos.

Esta relativa independización axiológica (Torregrosa, 1972, p. 42) se manifiesta en un «sistema propio y alternativo de valores» (Díez del Río, 1982). Pero es sobre todo un «rechazo de los valores adultos» (Arranz, 1982, p. 16), una "contestación de muchos valores y normas dominantes» (Zamora, 1993, p. 33). No en vano el sistema de valores adulto no es coherente (Reyzabal y Najt, 1982, p. 49), por lo que los jóvenes se negarán a asumir formas discutidas por los adultos (Beltrán y otros, 1984, p. 182). Pero no son solamente valores, los jóvenes cuestionan también las instituciones, pues éstas no se adaptan a ellos (Ander-Egg, 1980, p. 18). De este modo, los jóvenes rechazan el mundo en 
el que viven (Moya, 1983, p. 42) y muestran una clara voluntad de no caer en una sociedad extraña como la adulta (Arranz, 1982, p. 16).

De este modo, los jóvenes han de manifestar una importante rebeldía e inconformismo respecto de los adultos. Es lo que Griffin (1993) ha llamado el "discurso de la alienación» (disaffection), que sitúa en la desilusión juvenil con las instituciones de la sociedad adulta la causa de la rebeldía, si bien se diferencia entre el grado de rebeldía normal y la alienación que supone una amenaza para el orden social dominante (p. 201-202). Por su parte, la teoría subcultural señala que las subculturas juveniles expresan una resistencia y una oposición a problemas estructurales y a los valores de la clase media (Widdicombe y Wooffitt, 1995).

El discurso de la contestación parece especialmente diseñado para explicar los acontecimientos de los años sesenta, donde se explicitó el rechazo juvenil del mundo adulto y su apuesta por algo diferente. Pero todavía es posible encontrarlo para explicar ciertos comportamientos que resultan extraños o sin sentido: el gamberrismo y la violencia en general, el pasotismo, la cultura juvenil, etc. Esto es muestra de su buena salud, a pesar de estar enfrentado al discurso del narcisismo y el conformismo.

El discurso de la contestación se basa en la existencia de unos valores comunes a todos los jóvenes. Sin embargo, resulta complicado sostener la existencia de unos valores juveniles totalmente distintos de los de los adultos. La socialización ha de tener una impronta importante también a nivel axiológico. Como mucho se podría hablar de un diferente énfasis valorativo, mayor importancia de unos valores y menos de otros.

Este discurso tiene consecuencias similares a las que hemos señalado respecto al discurso del cambio (ver más arriba). Pero podríamos añadir una consecuencia positiva, que los adultos prestarán mayor atención a las inquietudes y propuestas de los jóvenes que emanan de estos valores positivos y no tanto a los problemas que plantean. Sin embargo, parece que finalmente el interés acaba por manifestarse en el intento de controlar las posibles instancias de rebeldía juvenil.

\section{La subcultura juvenil}

El discurso de la subcultura juvenil es también un lugar común en la literatura científica y tiene su origen en los estudios de la Escuela de Chicago (Thrasher, por ejemplo), que derivó en dos líneas de investigación, la subcultura delincuente y la subcultura juvenil como tal (Martín Criado, 1998). La juventud sería un colectivo con sus propias modas, preferencias, atuendos, valores, normas y símbolos (Torregrosa, 1972, p. 40) que definen un grupo homogéneo en cuanto a alternativas culturales (sistema de significados y modo de expresión y estilos de vida; Zamora, 1993, p. 34). Feixa (1998) remarca dos aspectos en la subcultura juvenil: los estilos de vida diferenciados y la formación de «microsociedades juveniles con grados significativos de autonomía respecto de las instituciones adultas» (p. 84). 
Se trata, pues, de una realidad diferenciada pero también heterogénea (García Rincón, 1994, p. 78). Así, la Escuela de Birmingham (Hargreaves, 1967; Willis, 1977) ha estudiado las subculturas juveniles —en plural- para referirse a los estilos juveniles más notorios por su atuendo o sus actuaciones y referirlas a la posición de clase de los jóvenes involucrados (Widdicombe y Wooffitt, 1995).

La subcultura juvenil aparece ante la divergencia en expectativas y valores normativos entre padres e hijos (Allerbeck y Rosenmayr, 1977, p. 97). Es una necesidad estructural de las sociedades modernas por la discontinuidad y falta de articulación en las pautas valorativas y los contenidos de roles (Torregrosa, 1972, p. 43). Así, la subcultura juvenil tiene una importante función adaptativa, esto es, permite a la juventud hacer frente a su situación transitoria (Gil Calvo y Menéndez, 1985, p. 27), da respuestas específicas a sus condiciones particulares (Zamora, 1993, p. 35). Por tanto, es la sociedad la que crea las condiciones del cambio, y convierte a la juventud en agente del mismo (Beltrán y otros, 1984, p. 183).

Como condición necesaria, la subcultura juvenil se basa en la «orientación obligatoria entre jóvenes» y en la «ligazón psíquica» que se crea entre ellos (Allerbeck y Rosenmayr, 1977, p. 94), fruto del importante tiempo que pasan juntos. Como efecto, la subcultura relativiza su recíproca dependencia de los estatus adultos (Torregrosa, 1972, p. 41).

En los años de la juventud la persona se forma con especial conciencia a través de sus actividades simbólicas (Willis, 1990, p. 7). Los jóvenes son muy dados al trabajo de desarrollar su propio estilo y decodificar los estilos de los demás en cuanto a música y orientaciones sociopolíticas. Así, los jóvenes pasan a formar parte de una subcultura, con un caudal de conocimientos y comportamientos compartidos en el grupo (Moore, 1994). Dentro de la subcultura juvenil se encuentran numerosas variaciones que en la literatura se vienen denominando «subculturas juveniles», pero también «estilos juveniles» o "tribus urbanas», realidades confluyentes aunque no equivalentes (ver Revilla, 1998). De hecho, es imprescindible tener en cuenta la diversidad entre los jóvenes, diferencias que vienen atravesadas por la estructura social, pues las subculturas reflejan las desigualdades sociales (ver Torregrosa, 1972; Gil Calvo y Menéndez, 1985). Algunos de estos estilos juveniles adquieren una notoriedad social muy importante, normalmente por los problemas que acarrean o por sus conductas «desordenadas». Lo significativo de estos grupos es que sus actuaciones se analizan como expresión extrema (vanguardia) de lo que la juventud tiene que decir (Widdicombe y Wooffitt, 1995, p. 21).

Este discurso acentúa sobremanera la diferencia entre jóvenes y adultos. Al enfatizar tal distinción se tiende a homogeneizar el interior, pensar a la subcultura juvenil como mucho menos diferenciada de lo que apuntan otros indicios. Pero, a pesar de pensarse como variaciones de la cultura dominante, son escasos los estudios que relacionan una determinada subcultura juvenil con los valores o significados del entorno donde se expresa. 


\section{La transición a la vida adulta}

Una definición muy común de la juventud la presenta como una transición entre niñez y vida adulta. Es un tiempo de espera en el que el joven progresivamente va adquiriendo las responsabilidades adultas: productiva, conyugal, doméstica y parental (Gil Calvo y Menéndez, 1985, p. 17). O también un proceso de emancipación que concluye cuando se cumplen estas condiciones: independencia económica, autoadministración de recursos, autonomía personal y hogar propio (Zárraga, 1985, p. 25). La segunda enumeración parece tener mayor precisión conceptual, pero la primera está más cerca de la experiencia vital de las personas que realizan una transición «normal» en sentido estadístico: trabajo y familia biparental heterosexual.

Desde este punto de vista, el elemento más importante para que la transición conduzca a buen puerto es la inserción sociolaboral, la obtención de un trabajo que provea de los recursos necesarios. De ahí, toda la investigación acerca de las diferentes trayectorias que siguen los jóvenes hasta acceder al mercado laboral, y con ello en la precariedad de algunos itinerarios (Casal, Masjoan y Planas, 1988). Es necesario conocer las estrategias y condiciones sociales que concurren en el paso a la edad adulta. Serrano (1995) incide sobre los distintos significados de la transición y el trabajo según la forma que toma la inserción sociolaboral. Galland (1984, 1991) observa dos modelos bien diferenciados de entrada en la vida adulta, el de la instalación -instantaneidad de los jóvenes de extracción obrera-y el de la postergación (différément) de los jóvenes burgueses a la espera de que la formación y el tiempo les proporcionen una posición social acorde a sus expectativas. Mientras que estos autores señalan de algún modo al sistema como responsable de la dificultad de la inserción laboral, Griffin (1993) constata la aparición en el ámbito anglosajón de planteamientos acerca de la inadecuación de los jóvenes al mercado laboral, sobre las condiciones de «empleabilidad» de los jóvenes para su integración social.

En este discurso el estatus juvenil se piensa como incompleto y parcialmente carente, pues al fin y al cabo el punto de mira se encuentra en el estatus adulto. Allerbeck y Rosenmayr (1977) hablan de los roles que pueden cumplir los jóvenes y aquéllos que les están prohibidos; Torregrosa (1972), de la ambivalencia del estatus juvenil. Zárraga (1985) incide sobre el desequilibrio entre capacidad y competencia, entre madurez y cualificación. Esta incompletud solamente puede ser superada con el ingreso de la persona en el mundo adulto, con lo que volvemos a la necesidad de la transición.

La presencia de este discurso en nuestra sociedad es innegable. La presión que supone el paro juvenil como problema es ineludible para cualquier joven. $Y$ es que el trabajo no puede dejar de ser el elemento fundamental en la inserción plena de cualquier persona en la sociedad.

Quizá el mayor problema de este discurso y de estas líneas de investigación sea que se han centrado demasiado en lo que es una transición normal, la más habitual, lo que supone dejar fuera a algunos colectivos sociales. Se 
prima la transición hacia una pareja heterosexual que formen un núcleo doméstico propio con su prole. No se ha dado buena cuenta tampoco de la situación diferencial de las mujeres. Además, con este discurso se pierde de vista lo específico de la juventud. No sabemos el papel que desempeñan las subculturas juveniles en la transición, tampoco si la inserción laboral informa todo el período juvenil o solamente momentos clave, cuando existe la posibilidad de un cambio de trayectoria. En definitiva, este discurso necesita ser complementado por otros que tengan que cuenta esos y otros aspectos.

\section{Juventud subordinada y discriminada}

Este discurso enfatiza las relaciones de poder, desiguales, entre jóvenes y adultos. Esto coloca a los primeros en una posición subordinada con la expectativa de unos beneficios futuros (González Blasco y otros, 1990, p. 14). Según Bourdieu (apud Martín Criado, 1998) y Sagrera (1992), se trata de la discriminación del grupo dominante adulto para proteger sus privilegios frente a las nuevas generaciones, y así se agravará en períodos donde hay mayor número de jóvenes y menor de adultos. En momentos como éste, aumenta la progresiva marginación de la juventud (Cembrano, 1986), pues el grupo dominado pierde su capacidad de acceder a los bienes.

De esta forma, el constructo «juventud» se convierte en un instrumento de dominación para los que lo aceptan para sí y se enorgullecen al apropiárselo (Sagrera, 1992, p. 30), pues la mitificación de lo juvenil conduce a los jóvenes a la aceptación gozosa de su estatus dominado. La relación de exclusión explica también el estereotipo negativo adulto sobre los jóvenes (Zamora, 1993, p. 31; Revilla, 1998), como elemento simbólico legitimador.

La mayor virtualidad de este discurso es poner de relieve una situación que dista mucho de ser igualitaria. Lo que la hace especialmente ambigua, en cualquier caso, es que se trata de una relación destinada a que desde ella misma se plantee su desaparición (Torregrosa, 1972, p. 50). Esto da lugar a un largo período de negociación más o menos violento, en el que unos pedirán mayores cotas de autonomía y otros se resistirán a concederla.

Pero, como señalan Bourdieu y Passeron (1970), es necesario observar diferencias entre la situación de unos y otros jóvenes frente a la reproducción social y a su toma del poder. Por un lado, las relaciones con los progenitores pueden acentuar o minimizar en lo posible la subordinación filial. Por otro, resulta difícil tratar conjuntamente la marginalidad de los jóvenes de clase baja y la subordinación harto beneficiosa de los jóvenes de clase alta respecto de sus padres, con todas las posibles situaciones intermedias que puedan imaginarse. De este modo, si bien cada joven se encuentra en posición de inferioridad respecto a los adultos de su entorno, las consecuencias de esa inferioridad distan mucho de ser equivalentes.

Por último, el discurso de la subordinación no está muy extendido en el medio social, pues se trata de un discurso potencialmente conflictivo y comprometedor. Circula más el discurso de la disolución de las relaciones de poder 
entre padres e hijos, el discurso de la permisividad, aireado por los medios de comunicación a partir de algunas investigaciones. Y si bien es difícil cuestionar la evolución que han sufrido las relaciones paterno-filiales en muy poco tiempo (Alberdi, 1999), no parece adecuado afirmar la desaparición total de la subordinación filial.

\section{La búsqueda de la identidad}

El principal impulsor de la relación entre juventud e identidad ha sido Erik H. Erikson $(1968,1972)$, para quien en este momento vital está en juego la adquisición de una identidad frente al peligro de quedar inmerso en difusión identitaria. La persona ha de integrar su pasado infantil y sus expectativas futuras durante un período de moratoria psicosocial, de libre experimentación de rol hasta que encuentre su propio lugar en la sociedad. El pensamiento de Erikson se ha extendido enormemente y ha convertido el discurso de la búsqueda de la identidad juvenil en moneda corriente. Es un discurso más querido para la psicología, pues es fácilmente integrable con su rama evolutiva, que convierte a la etapa juvenil en un momento más del desarrollo humano, lo que de algún modo naturaliza el proceso. También es un discurso muy presente en la psicología social, donde la identidad y la identidad social específicamente han sido objeto de estudio privilegiado (ver, por ej., Breakwell, 1992).

El discurso de la búsqueda de la identidad sitúa en la juventud la necesidad de encontrar una identidad propia (Arranz, 1982, p. 17). El período parecería caracterizable como un momento en el que se carece de identidad. Lozano i Soler (1994, p. 43) piensa que el principal problema de la juventud actual es la identidad, lo cual es aceptado conformadamente por los jóvenes. Y cuando se afirma la existencia de una identidad propia, se piensa como precaria (Feixa, 1989, p. 76). Como también son precarios los procesos generadores de identidad, los cuales conducen al fundamentalismo y la dispersión como opciones dominantes en lo que concierne a la identidad (Lozano i Soler, 1994, p. 44). Por otro lado, muchas explicaciones se apoyan en este discurso para explicar diversos fenómenos juveniles, especialmente la adscripción a subculturas o estilos.

No cabe duda de que entre la niñez y la etapa adulta la persona experimenta un gran cambio en su identidad. Pero este discurso tiende a enfatizar la crisis de la identidad juvenil y sobrevalorar la estabilidad y la falta de confusión entre los adultos, sin suficiente investigación que lo respalde.

Pero quizá fuera posible ofrecer un discurso alternativo sobre los cambios en la identidad. Cuando el niño se acerca al final de su período infantil, empieza ya a conocer lo que significa ser adolescente y joven, va accediendo a los materiales subculturales y a las pautas de actuación propias. Poco a poco irá actuando como tal adolescente o joven y se le irá reconociendo su nueva identidad, la identidad de joven. La persona se instalará confortablemente en su identidad juvenil durante un tiempo importante, lo que significa posicionarse más o menos cerca de unos y otros jóvenes. La música, la imagen y las actitudes sociopolíticas marcan las diferencias claves a este respecto. 
A lo largo del período juvenil, la persona irá también conociendo lo que significa ser adulto, las posibilidades de ser adulto que le son más o menos atractivas y más o menos accesibles. Así, irá tomando decisiones, más o menos conscientemente, que tendrán repercusión importante para su instalación en el mundo adulto: sobre su formación, su inserción laboral, su futura vida doméstica independiente (familiar o no), etc.

Con ello, el joven dejará de serlo paulatinamente, aunque ciertos acontecimientos marcarán puntos de inflexión importantes. La persona acabará ocupando una posición social y una identidad diferente que habrá dejado atrás en muchos aspectos a lo que fue su identidad de joven, pero en otros guardará similitudes. Pero la identidad personal seguirá sufriendo modificaciones y reelaboraciones a lo largo de la vida adulta de un modo distinto a lo que sucedió en el período juvenil.

Por supuesto, éste no deja de ser otro discurso sobre la identidad durante la juventud, pero es un discurso que nos parece más conveniente y plausible según el análisis que hemos realizado (ver Revilla, 1996 y 1998). Una virtualidad de este discurso es que presenta a los jóvenes no como personas carentes de identidad o en crisis constante, sino que los sitúa como individuos sujetos a una identidad suficientemente duradera como para ser tenida en cuenta independientemente de otras posibilidades de ser humanas.

\section{La diversidad juvenil}

El discurso de la diversidad juvenil tiene su rasgo principal en señalar la pluralidad e incluso la contradicción entre los mundos juveniles (Arranz, 1982, p. 11), la diversidad de estilos y desigualdad económica (Gil Calvo, 1985). La juventud es heterogénea desde el punto de vista de la clase social (Torregrosa, 1972, p. 27; Beltrán y otros, 1984, p. 201; Martín Criado, 1998, p. 15). La diversidad también se manifiesta en cuanto a las trayectorias de integración en la vida adulta (Casal, Masjoan y Planas, 1988; Agulló, 1996; Serrano, 1995).

La diversidad es palpable en la pluralidad de estilos juveniles, en el consumo diferencial, en las distintas actividades que se prefieren, etc. De este modo, sería posible hablar de la juventud como de unidades generacionales diversamente estructuradas (Allerbeck y Rosenmayr, 1977, p. 27). A pesar de ello, para los autores que utilizan este discurso no supone ningún problema lanzarse inmediatamente a realizar afirmaciones genéricas aplicables a toda la juventud. Como dice Arranz (1982, p. 12), «existen rasgos mayoritarios y destacables en la heterogénea realidad juvenil».

Sin embargo, este discurso llevado a un extremo pone en entredicho la totalidad de los discursos que hemos visto hasta el momento (ver Martín Criado, 1998). Gran cantidad de las afirmaciones que se realizan no tienen en cuenta esta diversidad. Como máximo serían aplicables a una supuesta mayoría de jóvenes, con lo que su utilidad se vería reducida. Otro rango de discursos parece dirigirse a un grupo de jóvenes que parece ser el más representativo. En este momento, este grupo hegemónico de jóvenes sería, como apuntan 
Beltrán y otros (1984) y Martín Criado (1998, p. 28), la juventud burguesa de clase media, especialmente si es estudiante.

El discurso de la diversidad juvenil pone en cuestión desde el discurso de la mitificación juvenil — se mitifica solamente lo que corresponde a una parte de la juventud-, hasta el discurso de la búsqueda de la identidad — no todos los jóvenes experimentarán problemas identitarios. Pero en especial este discurso deshace la posibilidad de hablar de la juventud como sujeto histórico y como agente de cambio social. Si solamente una parte de la juventud protagoniza movimientos sociales, entonces habríamos de preguntarnos acerca de la posición que ocupa ese grupo juvenil y cuáles son sus intereses. Por tanto, habría que identificar si son los jóvenes y qué jóvenes los que se encuentran en la vanguardia de la sociedad, en el vértice de la innovación.

\section{Organizando un discurso general sobre la juventud}

Todos estos discursos tienen la pretensión de describir la realidad juvenil en su conjunto aun cuando hemos visto los problemas asociados a muchos de ellos. Esto ha sido apreciado por muchos de los autores a los que nos hemos referido, quienes de hecho han utilizado argumentaciones provenientes de varios de los discursos que hemos identificado. Por ejemplo, Gil Calvo puede ser, entre otros, un ejemplo de un intento de integración fructífera de varios de los discursos que hemos señalado (ver Gil Calvo, 1985; Gil Calvo y Menéndez, 1985). Y así, en nuestro análisis a Gil Calvo le hemos incluido en el discurso de la transición a la vida adulta (juventud como tiempo de espera hasta la asunción de las responsabilidades adultas), en el de la subcultura juvenil (función adaptativa de la subcultura juvenil), en el de la juventud discriminada y subordinada (doble vínculo), así como en el de la pluralidad juvenil (desigualdad de estilos y desigualdad de recursos; competencia entre jóvenes). Pero, por supuesto, no es el único: encontramos intentos de integración interesantes y útiles en Arranz, Griffin, Allerbeck y Rosenmayr, Beltrán y otros, etc.

Precisamente por esto, resultaría en extremo interesante producir un discurso, uno más, sobre la juventud, y el papel que ocupa en nuestra sociedad. Para ello, seleccionaremos los argumentos de cada uno de los discursos que hemos descrito, matizándolos, negándolos o dándoles la vuelta para acercarlos a nuestra posición. Pero también utilizaremos los resultados provenientes de nuestras propias investigaciones (Revilla, 1996 y 1998; Fernández Villanueva y otros, 1997 y 1998). Este discurso se presenta como una propuesta sobre la que discutir con la intención de establecer una marco general desde el que pensar la juventud actual en nuestro país. Creemos que cualquier otra proposición que no tenga estas pretensiones ha de tratar de especificar la población a la que trata de analizar ${ }^{2}$.

2. Para aligerar el texto, y teniendo en cuenta que ya han sido citados previamente, evitaremos en estas páginas las referencias a autores concretos. 
La juventud es, ante todo, una población dependiente, subordinada y limitada en sus posibilidades de actuación respecto de los adultos. Pero no de los adultos en general, sino de aquéllos con los que interactúa habitualmente. Son estos adultos los que delimitan los márgenes dentro de los que se pueden mover los jóvenes y los beneficios - materiales y psicosociales - que pueden esperar de tal subordinación. En principio, en la medida en que las expectativas de beneficio sean mayores, el joven aceptará de mejor grado la relación asimétrica de poder.

La dominación se manifiesta en un estereotipo negativo que describe a los jóvenes como irresponsables, hedonistas, conformistas, pero que en otros momentos históricos podría girar en torno a otros significantes negativos. Esta construcción negativa se convierte en instrumento de control por parte de los adultos, pues los jóvenes que deseen integrarse en la sociedad deberán esforzarse en negarlo comportamentalmente, actuar de una manera que no pueda ser entendida en términos del estereotipo. A esto se une, en flagrante contradicción, un discurso positivo sobre la juventud como el mejor momento de la vida y la alta valoración de todo lo joven. Así, los jóvenes se adhieren con entusiasmo a su condición social, sin que dispongan del poder suficiente para cambiar el discurso negativo que circula sobre ellos.

Es también necesario reseñar que esta construcción cultural acerca de los jóvenes está más cerca de unos que de otros. Cuando se habla de juventud en general se tiende a referirse más a los estudiantes que a los trabajadores, más a los chicos que a las chicas, más a la clase media que a la baja. Se trata, pues, de un discurso normativo que dice lo que es normal y lo que no lo es dentro de los jóvenes.

Pero se trata de una relación de dependencia que ha de aligerarse y minimizarse inevitablemente según la persona va accediendo a la vida adulta. Un proceso difícil, con mayor o menor grado de violencia ${ }^{3}$, en el que se negocian los derechos que se le han de reconocer al joven y los deberes que se le pueden exigir, que cada vez han de ser mayores en ambos casos para acabar por convertirse en un actor social pleno.

Para salir de la dependencia es necesario acceder al estatus de adulto, lo que implica normalmente adquirir una independencia económica, normativa y de administración de recursos, así como la formación de un núcleo doméstico propio. Pero buena parte de la población no alcanza estas condiciones. Muchas mujeres pasan de depender de un padre a depender de un marido. La dependencia sobre los padres puede mantenerse parcialmente en los casos en los que se ha conseguido un empleo. Otros más no consiguen un trabajo suficiente como para mantenerse y ya han sobrepasado los límites convencionales que marcan su pertenencia a la juventud: no pueden ya ser considerados jóvenes y no han alcanzado plenamente el estatus adulto. Por tanto, debemos tener en cuenta la multitud de trayectorias hacia la vida adulta.

3. Violencia en el sentido desarrollado por Fernández Villanueva y otros (1998), como estado de cosas que para su mantenimiento o modificación necesita de amenaza explícita o implícita. 
Esto nos pone sobre la pista de que la juventud tiene en la edad un elemento importante. No se trata de que sea algo meramente biológico; es, sin duda alguna, una construcción cultural, si bien basada en la cuantificación del tiempo vivido, en la edad. Como toda identidad, el joven ha de ser reconocido en su condición por las personas con las que interactúa. Y eso implica, en primer lugar, una apariencia física, un cuerpo y una cara jóvenes. En nuestra sociedad, además, diversos documentos dan fe de nuestra edad, es también una de las primeras cosas que uno investiga cuando conoce a otra persona. El número de años vividos nos sitúa a cada individuo en una edad concreta, definida. Cuerpo y DNI son, pues, los dos elementos «biológicos» que conceden o niegan la identidad de joven según las convenciones sociales reinantes en cada momento histórico.

Los múltiples discursos que circulan sobre los jóvenes tematizan las cuestiones sobre las que tiene sentido reclamar una identidad de joven. Estos materiales son los que delimitan las posibilidades de ser joven, mediante la negación o afirmación de cada uno de estos discursos, y constituyen el marco genérico de la subcultura juvenil.

El joven, para serlo verdaderamente, habrá de situarse en relación con otros jóvenes, y así encontrar su identidad específica dentro de la juventud. Especialmente habrá de situarse respecto a los jóvenes más cercanos a él o a ella, su grupo de interacción, los amigos y los conocidos, pero también respecto a las imágenes juveniles que recibe de los medios de comunicación social. La identidad de joven no será algo estático e inamovible, como tampoco caótico y sin sentido. La persona irá añadiendo nuevos significados, abandonando otros, adscribiéndose a ciertos significantes, etc. Cuando se acerque el momento en que la persona nunca más será reconocida como joven, irá sustituyendo muchos significantes y significados identitarios por otros propios de adultos. Pero siempre quedará algo de lo que fue cuando joven, una marca que permita establecer una continuidad entre lo que fue y lo que es.

Las subculturas juveniles proveen a los jóvenes de gran cantidad de materiales con los que identificarse y construir su identidad. Por subculturas juveniles entendemos una serie de significados y modos de expresión comunes a una parte de la juventud que cristalizan en los diferentes estilos juveniles. Los estilos juveniles comprenden un estilo musical concreto, una imagen y un atuendo reconocible y una serie de actitudes sociopolíticas. Existen estilos juveniles más o menos conocidos, más o menos seguidos por los distintos jóvenes, mejor o peor definidos. Pero en cualquier caso, son susceptibles de ser utilizados por los jóvenes para construir su identidad. En algunas ocasiones, la adscripción al estilo es total, al menos durante un cierto tiempo. Pero en la mayoría cada joven se reconoce sólo parcialmente con el estilo, con alguno de sus componentes significativos: la música, parte del atuendo, alguna determinada actitud. Esta adscripción parcial permite a la persona conservar su especificidad identitaria, es una creación personal y no una mera copia de un estilo de moda. 
En principio, cada estilo juvenil y cada subcultura particular ha de ser fruto de un medio social determinado, posible por la existencia de una situación similar en un espectro juvenil concreto. Sin embargo, hay que tener en cuenta la difusión de materiales culturales e identitarios entre unos y otros grupos de jóvenes, influencias múltiples que tienen como marco posibilitador aquellos espacios que comparten jóvenes de distinta procedencia, así como los medios de comunicación que difunden selectivamente algunos estilos juveniles, normalmente los más problemáticos, sea cual sea la razón. Por ello es realmente complicado establecer una procedencia social clara de cada uno de los estilos juveniles, sin menoscabo de que futuras investigaciones puedan dar una respuesta satisfactoria.

La subcultura juvenil no se encuentra en oposición a la cultura de la sociedad en su conjunto. Podríamos decir que acentúa algunos aspectos y minimiza otros. Tampoco es una construcción total, sino que limita su grado de determinación a los aspectos que son relevantes para los diferentes discursos juveniles. En definitiva, se trata de una subcultura equiparable a las otras muchas variaciones de la cultura dominante, limitadas en su extensión a determinados ámbitos sociales. La particularidad de la subcultura juvenil es su extrema variedad de significados, derivada de la muy diferente posición de unos y otros jóvenes, pero que, a pesar de ello, han de enfrentar parecida construcción social acerca de lo que significa ser joven.

La juventud como conjunto no innova. Innovan los creadores, jóvenes o no, que por su posición social están en disposición de hacerlo y sin escapar nunca del marco social en el que se mueven. Pero la juventud sí tiene un papel claro respecto al cambio social. Los jóvenes, como recién llegados a la vida social, reciben lo que de nuevo se está produciendo en la sociedad de una manera rotunda. Recogen el momento presente como lo único existente, sin las cargas y sin la experiencia que proporciona el pasado. De este modo, la juventud está más cerca de la novedad, de la innovación. Se adhieren con fuerza a las corrientes del presente, si bien en la forma en que lo perciben desde su posición social concreta. Por ello la relación de cada grupo de jóvenes con el cambio social será específica y diferente. Pero podemos afirmar que los jóvenes son los que están más cerca del cambio dentro de cada ámbito social y que por ello lo condensan y facilitan gracias a su condición particular.

Con tal caracterización de la juventud ha debido quedar patente que no podemos pensar en la juventud ni como agente de cambio como tal ni como sujeto histórico. Solamente ciertos grupos de jóvenes tomarán un protagonismo importante cuando tengan una serie de intereses comunes por los que luchar y consigan organizarse en torno a unos objetivos concretos, sean éstos los que sean. Por tanto, esto probablemente pueda ocurrir desde los espacios en los que participan juntos ciertos tipos de jóvenes, como son el ámbito educativo y el de ocio. 


\section{Bibliografía}

AGULLÓ, E. (1996). Juventud, trabajo e identidad: la centralidad del trabajo en el proceso de construcción de la identidad de los jóvenes. Tesis doctoral. Madrid: Universidad Complutense de Madrid.

Alberdi, I. (1999). La nueva familia española. Madrid: Taurus.

Allerbeck, K.; Rosenmayr, L. (1977). Introducción a la sociología de la juventud. Buenos Aires: Kapelusz, 1979.

ANDER-EGG, E. (1980). La rebelión juvenil. Madrid: Marsiega.

ARANGUREN, J.L. (1982). Bajo el signo de la juventud. Madrid: Salvat.

ARIES, P. (1973). El niño y la vida familiar en el Antiguo Régimen. Madrid: Taurus. ARranz, E. (1982). «Análisis de la situación actual de la juventud». Documentación Social, 46, p. 11-26.

Arribas Macho, J.M.M.; GonZÁlez RodrígueZ, J.J. (1987). La juventud de los ochenta. Estudio sociológico de la juventud de Castilla y León. Valladolid: Junta de Castilla y León, Consejería de Educación y Cultura.

Beltrán, M.; García Ferrando, M.; González-Anleo, J.; López Pintor, R.; TOHARIA, J.J. (1984). Informe sociológico sobre la juventud española, 1960/82. Madrid: Ediciones SM.

Bourdieu, P.; PASSERON, J.C. (1970). La reproducción. Barcelona: Laia, 1977.

BREAKWELL, G.M. (ed.) (1992). Social Psychology of identity and the self-concept. Londres: Surrey University Press.

CASAL, J.; MaSjOAN, J.M.; Planas, J. (1988). «Elementos para un análisis sociológico de la transición a la vida adulta». Política y Sociedad, 1, p. 97-104.

Cembrano Díez, F. (1986). «La juventud y el cambio social». Documentación Social, 65, p. 165-180.

Corraliza, J.A. (1985). «Los jóvenes, ¿víctimas o amenaza?». Documentación Social, 58, p. $9-27$.

CRESPO, E. (1995). Introducción a la psicología social. Madrid: Universitas.

DíAz, A. (1989). "La caza del replicante». Alfoz, 62-63, p. 9-12.

DíEZ DEL Río, I. (1982). «La contracultura». Revista de Estudios de Juventud, 6, p. 101-132.

Dura Grimalt, R. (1987). «Juventud, rock \& roll y televisión». Revista de Estudios de Juventud, 28, p. 53-57.

ERIKSON, E.H. (1968). Identidad, juventud y crisis. Buenos Aires: Paidos, 1974.

- (1972). Sociedad y adolescencia. México: Siglo XXI, 1989.

FEIXA, C. (1989). «Hacia una antropología de la juventud urbana». Revista de Estudios de Juventud, 34, p. 69-78.

- (1998). De jóvenes, bandas y tribus. Barcelona: Ariel.

Fernández Villanueva, C.; Domínguez, R.; Revilla, J.C.; Guzmán, B. (1997). Estudio sociológico sobre la juventud de Leganés. Madrid. Mimeo.

Fernández Villanueva, C.; Domínguez, R.; Gimeno, L.; Revilla, J.C. (1998). Jóvenes violentos: causas psicosociológicas de la violencia de jóvenes en grupo. Barcelona: Icaria.

Galland, O. (1984). «Précarité et entrées dans la vie». Revue Française de Sociologie, XXV, p. 49-66.

- (1991). Sociologie de la jeunesse. L'entrée dans la vie. París: Armand-Colin.

GARCía RINCÓN, C. (1994). "¿Qué percepción tienen los jóvenes de esta sociedad?». Documentación Social, 95, p. 73-92.

Gil Calvo, E. (1985). Los depredadores audiovisuales. Madrid: Tecnos. 
Gil Calvo, E.; MenéNDeZ, E. (1985). Ocio y prácticas culturales de los jóvenes. Madrid: INJUVE.

GonzÁlez Blasco, P.; Andrés Orizo, F.; Toharia, J.J.; Elzo, J. (1990). Jóvenes españoles 89. Madrid: Ediciones SM. Fundación SM.

GRIFFIN, C. (1993). Representations of youth. The study of youth and adolescence in Britain and America. Cambridge: Polity Press.

HARGREAVES, D.H. (1967). Social Relations in a secondary school. Londres: Routledge and Kegan Paul.

LeVI, G.; SchmitT, J.-C. (1995). Historia de los jóvenes. Madrid: Taurus, 1996.

LOZANO I SOLER, J.M. (1994). «¿De qué hablamos cuando hablamos de los jóvenes?». Documentación Social, 95, p. 37-51.

Martín Criado, E. (1998). Producir la juventud. Crítica de la sociología de la juventud. Madrid: Istmo.

Martín Serrano, M. (1992). Historia de los cambios de mentalidades de los jóvenes entre 1960 y 1990. Madrid: Instituto de la Juventud.

MoOre, D. (1994). The Lads in action. Social processes in an urban youth subculture. Aldershot: Arena.

MosCovici, S. (1979). Psicología de las minorías activas. Madrid: Morata, 1981.

MOYA, C. (1983). «Informe sobre la juventud contemporánea». De juventud, 9, p. 25-52.

ReVILLA, J.C. (1996). La identidad personal en la pluralidad de sus relatos: estudio sobre jóvenes. Tesis doctoral. Madrid: Universidad Complutense de Madrid.

- (1998). La identidad personal de los jóvenes: pluralidad y autenticidad. Madrid: Entinema.

Reyzabal, M.V.; NajT, M. (1982). "Conflicto e identidad de la juventud, hoy». Documentación Social, 46, p. 45-56.

SÁEZ MARÍn, J. (1995). «Los estudios sobre juventud en España: contextos de un proceso de investigación-acción (1960-1990)». Revista Internacional de Sociología, 10, p. 159-197.

SAGRERA, M. (1992). El edadismo: contra "jóvenes» y «viejos», la discriminación universal. Madrid: Fundamentos.

SERrano, A. (1995). «Diversificación de las trayectorias de inserción sociolaboral». En González, L.; Torre, A. de la; Elena, Joaquín de (comps.). Psicología del trabajo y de las organizaciones, gestión de los recursos humanos y nuevas tecnologias, p. 455-462. Salamanca: V Congreso Nacional de Psicología Social.

Torregrosa, J.R. (1972). La juventud española. Conciencia generacional y politica. Barcelona: Ariel.

VAllés MarTínez, M. (1989). Abrirse camino en la vida: proyectos vitales de los jóvenes madrileños. Tesis doctoral. Madrid: Universidad Complutense de Madrid.

Widdicombe, S.; WoOfFiTT, R. (1995). The language of youth subcultures. Social identities in action. Hemel Hempstead: Harvester Wheatsheaf.

WILLIS, P. (1977). Learning to labour: how working class kids get working class jobs. Farnborough: Saxon House.

- (1990). Common culture. Symbolic Work at play in the everyday cultures of the young. Milton Keynes: Open University Press.

Zamora Acosta, E. (1993). Jóvenes andaluces de los 90. Sevilla: Junta de Andalucía, Escuela Pública de Animación Sociocultural.

ZÁRRAGA, J.L. de (1985). Informe Juventud en España. La inserción de los jóvenes en la sociedad. Madrid: Instituto de la Juventud. 